\title{
Dynamic Behaviors in Coupled Neuron System with the Excitatory and Inhibitory Autapse under Electromagnetic Induction
}

\author{
Ying Xu, ${ }^{1}$ Ya Jia ${ }^{D},{ }^{1}$ John Billy Kirunda, ${ }^{1}$ Jian Shen, ${ }^{1,2}$ Mengyan Ge, ${ }^{1}$ Lulu Lu, \\ and Qiming Pei ${ }^{3}$ \\ ${ }^{1}$ Institute of Biophysics and Department of Physics, Central China Normal University, Wuhan 430079, China \\ ${ }^{2}$ College of Electronics and Information Engineering, South-Central University for Nationalities, Wuhan 430074, China \\ ${ }^{3}$ School of Physics and Optoelectronic Engineering, Yangtze University, Jingzhou 434023, China
}

Correspondence should be addressed to Ya Jia; jiay@mail.ccnu.edu.cn

Received 4 April 2018; Revised 3 June 2018; Accepted 25 June 2018; Published 26 July 2018

Academic Editor: Viet-Thanh Pham

Copyright $\odot 2018$ Ying Xu et al. This is an open access article distributed under the Creative Commons Attribution License, which
permits unrestricted use, distribution, and reproduction in any medium, provided the original work is properly cited.

\begin{abstract}
The induced current produced by electromagnetic induction can adjust the membrane potential of neuron through the feedback of a magnetic flux-controlled memristor. We adopt the numerical simulation method with the aim of investigating the synchronous behavior in the neuronal system that is coupled by chemical and electrical synapses under electromagnetic induction. Within the improved model, the effects of electromagnetic induction on neurons are described with additive memristive current on the membrane variable, and the memristive current is dependent on the variation of magnetic flow. The simulation results show that the two coupling modes play an important role in the synchronization of the system. By increasing the chemical synaptic feedback gain, we observe a transition from mixed oscillatory to periodic state at a critical value. In addition, two Hopf bifurcation points are found with the change of the external stimuli, and the state of neuron discharge is influenced by initial values. Furthermore, there is a domain of coupling strength and feedback gain values, in which the two coupled neuron system is synchronized and longer time lag is not conducive to the system synchronization.
\end{abstract}

\section{Introduction}

A neural system, which is made up of a large number of neurons, is a complex information network. Different types of discharge patterns can be switched under the control of external stimulation or bifurcation parameter. In order to understand the regulating function of the nervous system, many models of neuronal electrical activity have been proposed. Commonly used models include the FitzHugh-Naguma model [1], Morris-Lecar neuron model [2], Hindmarsh-Rose model [3, 4], Nagumo-Sato neuron model [5], and Wilson-Cowan neuron model [6]. These models that describe neuron dynamics with a set of differential equations are almost derived from the Hodgkin-Huxley [7] model or the simplified version. Some results from biological experiments [8-10] can be explained by theoretical neuron models, such as the MorrisLecar neuron model. In this model, the membrane potential exhibits quiescent, spiking, or bursting state by changing the external forced current [11]. Neurons do not work in isolation, but they interact to affect the processing of information. There are two forms of synaptic coupling found in the real nervous system, namely, electrical synapse and chemical synapse. The synchronization phenomena are a typical manifestation of the rhythms of group movement; that is, all neurons in the system have a certain connection at the same time or rhythm [12-14]. Bazhenov et al. [15] designed a coupled linear chain of Hindmarsh-Rose model neurons with reciprocal inhibition between neighboring neurons that exhibited synchronous oscillations. Zhang et al. [16] proposed a class of synchronization problems of nonlinear time-delay dynamic networks with a nonuniform impulse effect. Burić et al. [17] studied the synchronization of Hindmarsh-Rose neurons with a time-delayed fast threshold modulation synapse. $\mathrm{Xu}$ et al. [18] analyzed the synchronization behavior and mode selection in neural networks under the coupling of chemical or electrical synapses. Yao et al. [19] investigated the influence of 
coupling strength, time delay, and network topology on synchronization behavior in delay-coupled networks of chaotic pendulums. Gokul and Kapitaniak [20] studied the synchrony of coupling multistable systems which have hidden attractors with each other. In coupled oscillators or coupled neurons, synchronization may occur because of the appropriate coupling effect [21-24]. Interestingly, the stochastic and coherence resonance [25-27] of the nervous system is induced by appropriate noise intensity and external periodic stimulus. The synchronization of the coupling system is an interesting research filed. It is challenging to analyze the dynamic mechanism caused by the variation of the coupling parameters and modes of the system. The synchronization phenomena in Hindmarsh-Rose (HR) neurons that are connected by electrical coupling and chemical coupling, moreover, complete synchronization, phase synchrony, and antisynchrony of neurons are realized [28, 29]. The neural electrical activity has also been widely studied and verified in the circuit [30-35]. For example, Vaidyanathan et al. [36-38] designed electronic circuits to study the feasibility of the 3D novel jerk chaotic system with hyperbolic sinusoidal nonlinearity. Conti and Turchetti [39] performed a circuit to realize approximate identity neural network for the analog synthesis nonlinear dynamical system. Pham et al. [40] proved the existence of chaotic behavior in a threedimensional autonomous chaotic system with a circular equilibrium by using OrCAD PSpice software and experimental.

It is necessary to study the effects of electromagnetic induction on neuronal cells [41-44]. The changes of membrane potential can induce electromagnetic induction between neurons. As reviewed in $[45,46]$, the effects of electromagnetic radiation in Homo sapiens include electrical activity of neurons, energy metabolism, genomic responses, neurotransmitter balance, blood-brain barrier permeability, cognitive function, sleep, brain tumors, and other encephalopathy. Lu et al. [47-49] investigated the effects of highand low-frequency signal stimulus on neural activity under electromagnetic radiation. According to Faraday's law of induction, the magnetic field is a result of fluctuations in the action potential. That is, the distribution of electromagnetic field both inside and outside neurons can be changed by the fluctuation of the membrane potential. Therefore, a new three-variable ML neuron model is established by introducing an additional variable as magnetic flux which adjusts the membrane potential via a memristor $[50,51]$.

The following study is based on the proposed MorrisLecar neuron model with consideration of magnetic flux, in which the dynamic characteristics of the neurons are studied by using bifurcation diagrams and time series of the discharge. A preliminary synchronization analysis was conducted in the excitatory and inhibitory neural system. The study revealed that excitatory and inhibitory neurons can be synchronized under the appropriate coupling strength. The synchronization behavior of the system is also affected by the time lag when the coupling strength and the feedback gain are maintained.

\section{Model and Scheme}

The Morris-Lecar (ML) equations were originally developed as a mathematical model of muscle fiber. For the neuron, the effect of electromagnetic induction should be considered during the discharge process of the membrane potential. The electric activity will change because of the fluctuation of electromagnetic induction and ion concentration in the process of ion exchange. We modify the basic ML model, including the impact of the electromagnetic radiation. The improved ML neuronal model [44] contains three variables, and the dynamic properties are described as follows:

$$
\begin{aligned}
c \frac{d V}{d t}= & g_{\mathrm{Ca}} m_{\infty}(V)\left(V_{\mathrm{Ca}}-V\right)+g_{\mathrm{K}} \omega\left(V_{\mathrm{K}}-V\right)+g_{\mathrm{L}}\left(V_{\mathrm{L}}-V\right) \\
& -k \rho(\varphi) V+I_{\mathrm{ext}}, \\
\frac{d \omega}{d t}= & \lambda_{\omega}(V)\left(\omega_{\infty}(V)-\omega\right), \\
\frac{d \varphi}{d t}= & k_{1} V-k_{2} \varphi,
\end{aligned}
$$

with

$$
\begin{aligned}
& m_{\infty}(V)=0.5+0.5 \tanh \left(\frac{V-V_{1}}{V_{2}}\right), \\
& \omega_{\infty}(V)=0.5+0.5 \tanh \left(\frac{V-V_{3}}{V_{4}}\right) \\
& \lambda_{\infty}(V)=\phi \cosh \left(\frac{V-V_{3}}{2 V_{4}}\right)
\end{aligned}
$$

where $V$ and $\omega$ denote the variables for the membrane potential $(\mathrm{mV})$ and gate channel, respectively. Parameter $c$ is the capacitance of the membrane $\left(\mu \mathrm{F} / \mathrm{cm}^{2}\right)$. The $g_{\mathrm{Ca}}, g_{\mathrm{K}}$, and $g_{\mathrm{L}}$ denote the maximum conductance $(\mathrm{mS})$ of calcium ion, potassium ion, and leak ion, respectively. $V_{\mathrm{Ca}}, V_{\mathrm{K}}$, and $V_{\mathrm{L}}$ are the reversal potential $(\mathrm{mV})$ corresponding to these channels. $m_{\infty}(V)$ and $\omega_{\infty}(V)$ define the value of the opening probability for the calcium ion channel and the potassium ion channel in the steady state, where $V_{1}, V_{2}, V_{3}$, and $V_{4}$ are the parameters of the steady system, and $\lambda_{\omega}(V)$ defines the rate constant for the opening of potassium ion channel. The parameter $\phi$ is marked as the variation between the fast and the slow scales of neurons.

As described in $[52,53]$, the variations of the intercellular and extracellular ion concentration can induce electromagnetic induction, which can be expressed by magnetic flux according to Faraday's law of electromagnetic induction. The induced current produced by electromagnetic induction can adjust the membrane potential by the feedback of the memristor. The memristor in model (1) can be divided into two ways: the charge controlled and the magnetic controlled. For the potassium ion-channel memristor, the second term in the right of (1) can be rewritten as $i_{\mathrm{K}}=G_{\mathrm{K}}(\omega) v_{\mathrm{K}}$ with $v_{\mathrm{K}}$ $\Leftrightarrow V-V_{\mathrm{K}}$ and $G_{\mathrm{K}} \Leftrightarrow g_{\mathrm{K}} \omega$, and $G_{\mathrm{K}}$ is the potassium memductance function. The fourth term in the right of (1) can be rewritten as $i_{\varphi}=G_{\varphi}(\varphi) v_{\varphi}$ with $v_{\varphi} \Leftrightarrow V$ and $G_{\varphi} \Leftrightarrow k \rho(\varphi)$, which defines another first-order memristor, and the conductance value of the memristor depends on the input current. The expression of $\rho(\varphi)=\alpha+3 \beta \varphi^{2}$ denotes the memory conductance of a magnetic flux-controlled memristor [54], it is used to calculate the effect of feedback regulation on the 
membrane potential when the magnetic flux is changed, and $\alpha$ and $\beta$ are fixed parameters. Therefore, as in [54], the induced current and electromagnetic induction can be described by

$i^{\prime}=\frac{d q(\varphi)}{d t}=\frac{d q(\varphi)}{d \varphi} \frac{d \varphi}{d t}=\rho(\varphi) \frac{d \varphi}{d t}=\frac{d \varphi}{d t}\left(\alpha+3 \beta \varphi^{2}\right), \quad \frac{d \varphi}{d t}=k V$.

The variable $i^{\prime}$ represents induction current. The term $k \rho(\varphi) V$ represents the inhibitory modulation of membrane potential, and it describes the induced current induced by electromagnetic induction. The parameter $k$ is the induction coefficient, and its value depends mainly on the medium itself. $I_{\text {ext }}$ is the external forcing current. The terms $k_{1} x$ and $k_{2} \varphi$ in the (1) mean the influence of membrane potential on magnetic flux and leakage of magnetic flux, respectively.

For the analysis of the possibility and stability of the synchronized dynamics between two neurons under bidirectional coupling, the dynamic equations are given by

$$
\begin{aligned}
c \frac{d V_{\alpha, \beta}}{d t}= & g_{\mathrm{Ca}} m_{\infty}\left(V_{\alpha, \beta}\right)\left(V_{\mathrm{Ca}}-V_{\alpha, \beta}\right)+g_{\mathrm{K}} \omega_{\alpha, \beta}\left(V_{\mathrm{K}}-V_{\alpha, \beta}\right) \\
& +g_{\mathrm{L}}\left(V_{\mathrm{L}}-V_{\alpha, \beta}\right)-k \rho\left(\varphi_{\alpha, \beta}\right) V_{\alpha, \beta}+I_{\text {ext }}+I_{\text {syn }} \\
& +C\left(V_{\alpha, \beta}-V_{\beta, \alpha}\right), \\
\frac{d \omega_{\alpha, \beta}}{d t}= & \lambda_{\omega}\left(V_{\alpha, \beta}\right)\left(\omega_{\infty}\left(V_{\alpha, \beta}\right)-\omega_{\alpha, \beta}\right), \\
\frac{d \varphi_{\alpha, \beta}}{d t}= & k_{1} V_{\alpha, \beta}-k_{2} \varphi_{\alpha, \beta},
\end{aligned}
$$

where the subscripts $\alpha$ and $\beta$ are a pair of coupled ML neurons under electromagnetic radiation. $C$ denotes coupling intensity between adjacent neurons.

In order to simulate the chemical synapse feedback of neurons, we shall use the so-called fast threshold modulation scheme proposed by Somers and Kopell [55] and often used by others, for example, [56, 57]. This chemically feedback form, which clearly combines the time lag of the synapse, is provided by the following functions:

$$
I_{\text {syn }}=-\frac{H_{\text {syn }}\left(V_{\alpha \beta}-V_{\text {syn }}\right)}{1+\exp \left[-\sigma\left(V_{\alpha \beta}(t-\tau)-\theta\right)\right]} .
$$

The variable parameter $H_{\text {syn }}$ is the feedback gain at time $t$ with itself connected at time $t-\tau$. The symbol $\tau$ indicates the time lag (ms) of the signal propagation. $C$ is coupling strength between two neurons. $V_{\text {syn }}$ represents the synaptic reversal potential $(\mathrm{mV})$, which depends on the presynaptic neurons and receiver. The chemical coupling is characterized by the difference between the synaptic reversal potential and the synaptic potential. A positive or negative sign of the difference corresponds to an excitatory or inhibitory effect of the synapse. If the synapsis is excitatory, $V_{\text {syn }}=15 \mathrm{mV}$, and if the synapsis is inhibitory, $V_{\text {syn }}=-10 \mathrm{mV}$. The parameter $\theta$ is a synaptic threshold. Considering that the neuron membrane potential value of the improved $\mathrm{ML}$ model is between $-17 \mathrm{mV}$ and $15 \mathrm{mV}, \theta=4 \mathrm{mV}$ is selected to ensure that the spike of the $V$ is over the threshold, and the quiescent state of the $V$ is less than the threshold. That is, the membrane potential of the presynaptic neuron is more than $\theta$, and it can play a role in the postsynaptic neuron $[58,59]$. $\sigma$ is the ratio constant to the start of excitement or inhibition. In this paper, we focus on the collective behavior of the two coupled neuron system driven by the excitatory and inhibitory autapse, and the schematic diagram is shown in Figure 1. Parameters of the improved ML neuronal model are given as $c=20 \mu \mathrm{F}, V_{\mathrm{Ca}}=120 \mathrm{mV}, V_{\mathrm{K}}=-84 \mathrm{mV}$, $V_{\mathrm{L}}=-60 \mathrm{mV}, \quad g_{\mathrm{Ca}}=4 \mathrm{mS}, \quad g_{\mathrm{K}}=8 \mathrm{mS}, \quad g_{\mathrm{L}}=2 \mathrm{mS}, \quad V_{1}=$ $-1.2 \mathrm{mV}, V_{2}=18 \mathrm{mV}, V_{3}=12 \mathrm{mV}, V_{4}=17.4 \mathrm{mV}, \phi=0.067$, $k_{1}=0.1, k_{2}=0.01$, and $\sigma=-1$.

To characterize the synchronization in the system of coupled spiking neurons, a method of calculating the error function is introduced in the following [24]:

$$
e=\sqrt{\left(V_{\alpha}-V_{\beta}\right)^{2}+\left(\omega_{\alpha}-\omega_{\beta}\right)^{2}+\left(\varphi_{\alpha}-\varphi_{\beta}\right)^{2}}
$$

Equation (6) indicates that the lower the value of $e$ corresponds with the better synchronization in the system.

\section{Results and Discussion}

In this section, first of all, the bifurcation is theoretically analyzed to reveal the dynamic mechanism of the discharge mode in the improved model (1). Then the fourth-order Runge-Kutta method is used to calculate the improved ML neuronal model, the step of time $h$ is selected as 0.01 , and the transient period for calculating is 5000 time units.

Let

$$
\begin{aligned}
& \frac{d V}{d t}=f(V, \omega, \varphi), \\
& \frac{d \omega}{d t}=g(V, \omega, \varphi), \\
& \frac{d \varphi}{d t}=h(V, \omega, \varphi),
\end{aligned}
$$

and $\left(V_{s}, \omega_{s}, \varphi_{s}\right)$ is the equilibrium point; that is,

$$
f\left(V_{s}, \omega_{s}, \varphi_{s}\right)=g\left(V_{s}, \omega_{s}, \varphi_{s}\right)=h\left(V_{s}, \omega_{s}, \varphi_{s}\right)=0
$$

If the parameter $k=0.1$, the system (8) can be written in the form

$$
\begin{gathered}
4 m_{\infty}\left(V_{s}\right)\left(120-V_{s}\right)+8 \omega_{s}\left(-84-V_{s}\right)+2\left(-60-V_{s}\right)-0.1(0.1 \\
\left.+0.03 \varphi_{s}^{2}\right) V_{s}+I_{\text {ext }}=0 \\
\omega_{s}=\omega_{\infty}=0.5+0.5 \tanh \left(\frac{V_{s}+12}{17.4}\right) \\
\varphi_{s}=10 V_{s},
\end{gathered}
$$




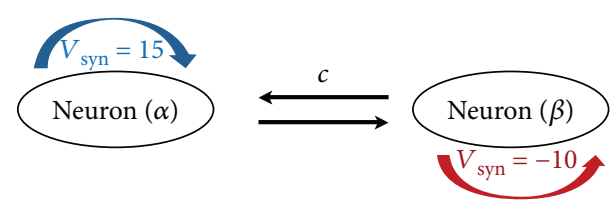

FIgURE 1: A schematic diagram of the coupled neuron system.

and thus,

$$
\begin{gathered}
2\left(1+\tanh \frac{V_{s}+1.2}{18}\right)-4\left(1+\tanh \frac{V_{s}-12}{17.4}\right)-2\left(60+V_{s}\right) \\
-0.1 V_{s}\left(0.1+3 V_{s}^{2}\right)+I_{\text {ext }}=0 .
\end{gathered}
$$

Obviously, the expression of the equilibrium point $\left(V_{s}, \omega_{s}, \varphi_{s}\right)$ can be obtained by (10). Then we analyze the stability of the equilibrium point with $I_{\text {ext }}$ as the bifurcation parameter. It is noted that the stability of the equilibrium point is determined by the eigenvalue of its Jacob matrix; that is, when all the eigenvalues are a negative real part, then the equilibrium point is stable; otherwise, it may be marginally stable or unstable [60]. The appearance of a pair of pure imaginary eigenvalues signifies Hopf bifurcation [61].

At the equilibrium point $\left(V_{s}, \omega_{s}, \varphi_{s}\right)$, the linearization Jacobi matrix of the improved model is that

$$
\mathbf{J}\left(V_{s}, \omega_{s}, \varphi_{s}\right)=\left[\begin{array}{ccc}
\frac{1}{c}\left[-4 m_{\infty} V_{s}+\left(480-4 V_{s}\right) \frac{\partial m_{\infty}}{\partial V}-8 \omega_{s}-2.01-0.003 \varphi_{s}^{2}\right] & \frac{8}{c}\left(84+V_{s}\right) & -0.006 \frac{V_{s} \varphi_{s}}{c} \\
\left(\omega_{\infty}-\omega_{s}\right) \frac{\partial \lambda_{\infty}}{\partial V}+\lambda_{\infty} \frac{\partial m_{\infty}}{\partial V} & -\lambda_{\infty} & 0 \\
0.1 & 0 & 0.01
\end{array}\right] .
$$

Therefore, the characteristic determinant of this system at the equilibrium point is

$$
\left|\mathbf{J}\left(V_{s}, \omega_{s}, \varphi_{s}\right)-\lambda I\right|=\left|\begin{array}{ccc}
\frac{1}{c}\left[-4 m_{\infty} V_{s}+\left(480-4 V_{s}\right) \frac{\partial m_{\infty}}{\partial V}-8 \omega_{s}-2.01-0.003 \varphi_{s}^{2}\right]-\lambda & -\frac{8}{c}\left(84+V_{s}\right) & -0.006 \frac{V_{s} \varphi_{s}}{c} \\
\left(\omega_{\infty}-\omega_{s}\right) \frac{\partial \lambda_{\infty}}{\partial V}+\lambda_{\infty} \frac{\partial m_{\infty}}{\partial V} & -\lambda_{\infty}-\lambda & 0 \\
0.1 & 0 & 0.01-\lambda
\end{array}\right|=0,
$$

where

$$
\begin{aligned}
\frac{\partial m_{\infty}}{\partial V} & =\frac{19}{36}-\frac{1}{2} \tanh ^{2}\left(\frac{V_{s}+1.2}{18}\right), \\
\frac{\partial \omega_{\infty}}{\partial V} & =\frac{46}{87}-\frac{1}{2} \tanh ^{2}\left(\frac{V_{s}-12}{17.4}\right), \\
\frac{\partial \omega_{\infty}}{\partial V} & =\frac{46}{87}-\frac{1}{2} \tanh ^{2}\left(\frac{V_{s}-12}{17.4}\right) .
\end{aligned}
$$

If the Jacobi matrix has one eigenvalue of the negative real part and two zero real parts at a critical value of the bifurcation parameter, then we usually say that a Hopf bifurcation occurs [61]. According to the relationship between $\lambda$ and $I_{\text {ext }}$, we calculate the branch of the equilibrium point which undergoes two Hopf bifurcations at parameter $I_{\text {ext1 }}=-27.99$ and $I_{\text {ext } 2}=23.79$. Obviously, they correspond to the bifurcation points HB in Figure 2(b). Similarly, the corresponding bifurcation points can be obtained by changing the parameter $k$.

The results of the bifurcation analysis for the system with the electromagnetic effect described by (1) are numerically simulated. In the bifurcation analysis, the influence of the external forcing current $I_{\text {ext }}$ on the discharge behavior of the neuron under different electromagnetic effects known as induction coefficient $k$ is investigated. The system will have the process of "resting-exciting-silent" by increasing the external forcing current. In Figure 2, several typical bifurcation diagrams for the different external forcing currents, without $(k=0)$ and with $(k \neq 0)$ the electromagnetic effects, are plotted, respectively.

Figure 2 shows the bifurcation with $I_{\text {ext }}$ as the bifurcation parameter for six different values of the induction coefficient. The equilibrium point of the system has undergone five changes without electromagnetic radiation, as shown in Figure 2(a). In the beginning, the system has only a stable equilibrium point, and the action potential of the neuron will 


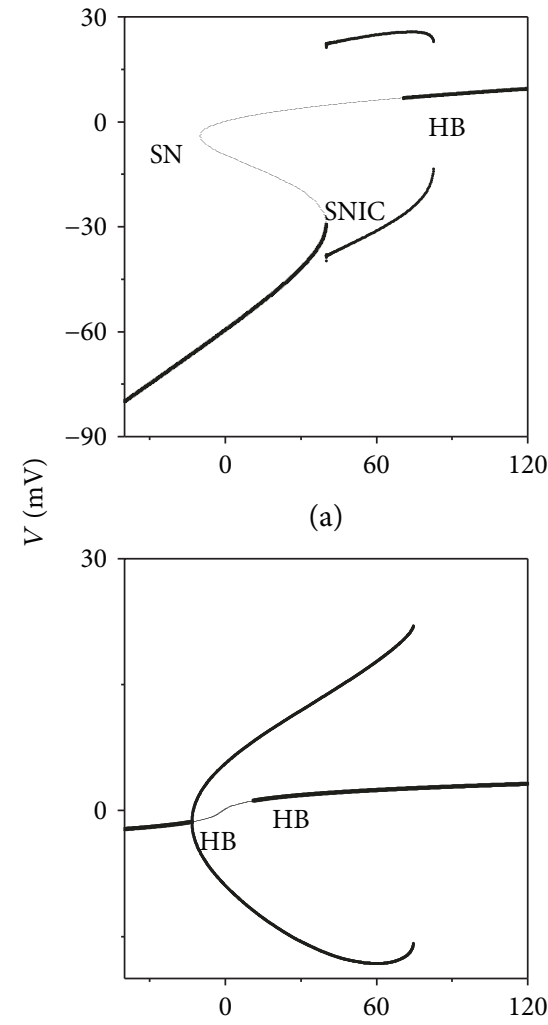

(a)

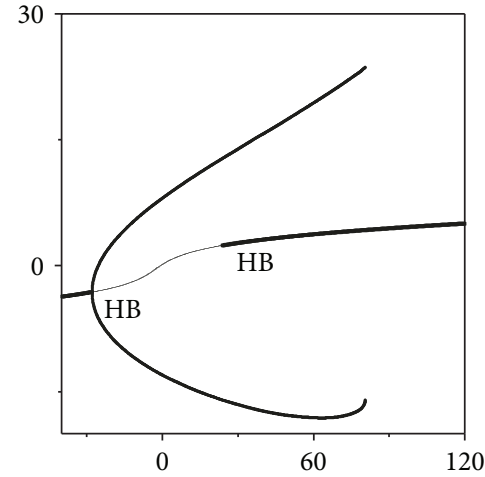

(b)

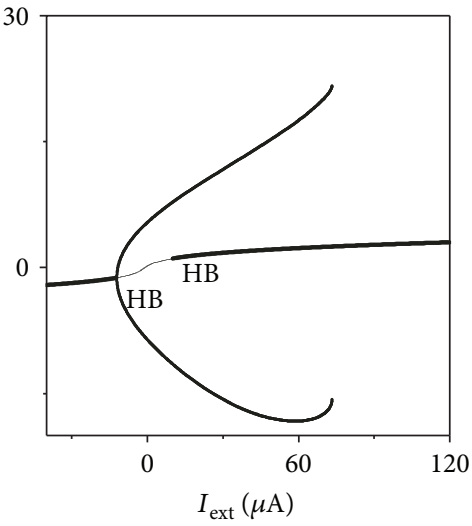

(b)

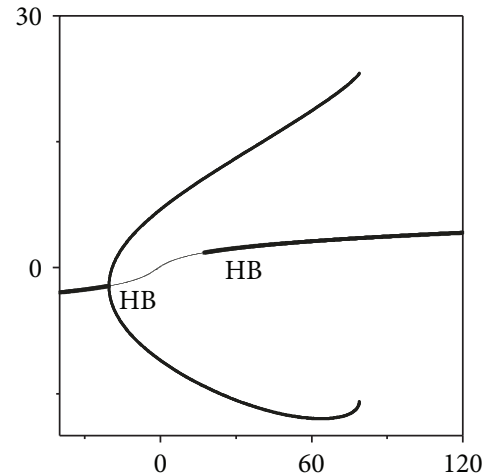

(c)

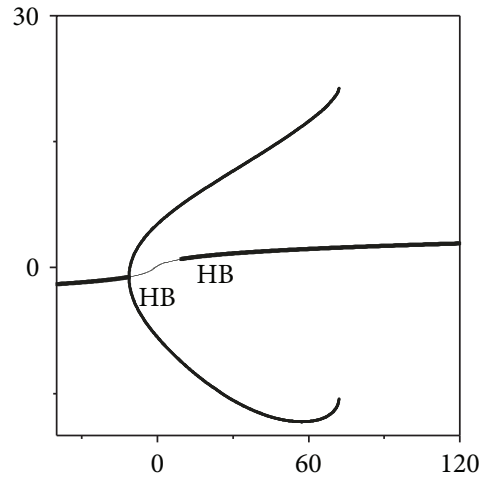

(c)

Figure 2: The bifurcation diagrams of $V_{\max }$ and $V_{\min }$ for the improved ML neuronal model driven by an external forcing current at $k_{1}=0.1$ and $k_{2}=0.01$; for (a) $k=0$, (b) $k=0.1$, (c) $k=0.2$, (d) $k=0.4$, (e) $k=0.5$, and (f) $k=0.6$.

eventually converge to a fixed value. When $I_{\text {ext }}>-9.93 \mu \mathrm{A}$, there exist three equilibrium points, of which two are unstable, and the action potential of the neuron will still converge to a fixed value. As the external current increases, the excitability of neurons in the saddle node bifurcation threshold is obtained, the threshold is $I_{\mathrm{ext}}=40 \mu \mathrm{A}$, and at this time, the membrane potential of the neuron is periodic discharge. On further increased $I_{\text {ext }}$, bistable state can be generated at $I_{\text {ext }}=70.4 \mu \mathrm{A}$, which corresponds to a subcritical Hopf bifurcation point. The limit cycle vanishes $I_{\text {ext }}=82.5 \mu \mathrm{A}$, and the system has only a stable state. The properties and positions of the bifurcation points have changed thoroughly, considering the neuron system after the electromagnetic effect, and the results are found in Figures 2(b)-2(f). There are two Hopf bifurcation points of the ML neuron system with the electromagnetic effect. The results in Figure 3(b) confirm the Hopf bifurcation points at $I_{\text {ext }}=-28.48 \mu \mathrm{A}$ and $23.55 \mu \mathrm{A}$. As the induction coefficient $k$ increases, a similar phenomenon is observed and the corresponding bifurcations are summarized in Table 1.

It can be noted from Table 1 that parameters describe the interaction between membrane potential and magnetic flux which is further increased to $k=0.6$, the threshold of excitability is increased to a higher value corresponding to $I_{\text {ext }}=$ $-11.39 \mu \mathrm{A}$ (see Figure 1 and Table 1). However, the position of the second bifurcation point was reduced from $23.55 \mathrm{mV}$ to $9.0 \mathrm{mV}$. That is to say, with the increase of the induction coefficient $k$, the region of the limit cycle in the system is gradually compressed. It should be pointed out that the system has a Hopf bifurcation point HOPF1 with a negative value of parameter $I_{\text {ext }}$ (see Table 1), which will not be considered in the following text since it loses the biophysical meaning. At the same time, in the numerical simulation, we find that when $I_{\text {ext }}>\mathrm{HOPF} 2$, the selection of the initial value of the system is as important as the external current. Based on the analysis of the bifurcation diagram of $V_{\max }$ and $V_{\text {min }}$ for the improved ML neuronal model, the external forcing currents $I_{\text {ext }}=40 \mu \mathrm{A}, I_{\text {ext }}=50 \mu \mathrm{A}, I_{\text {ext }}=60 \mu \mathrm{A}, I_{\text {ext }}=70 \mu \mathrm{A}$, and $I_{\text {ext }}=80 \mu \mathrm{A}$ are chosen to impose on the neuron. The type of membrane potential discharge at different initial values is calculated in Figure 3.

The results in Figure 3 show that the initial values of $V_{0}$ and $\omega_{0}$ have a great influence on the discharge of neurons in the improved ML model. The oscillating areas of the membrane potential are reduced by the increase of the external stimulation current; that is, the red area in the picture becomes smaller. The oscillating region of the membrane potential is found to be banded, which indicates the changes in the initial value of $\varphi_{0}$ with little effects on the discharge of neurons as shown in Figure 3, a2-e2 and a3-e3.

The results in Figure 4 show that the initial value region that produces the oscillations of the membrane potential is 

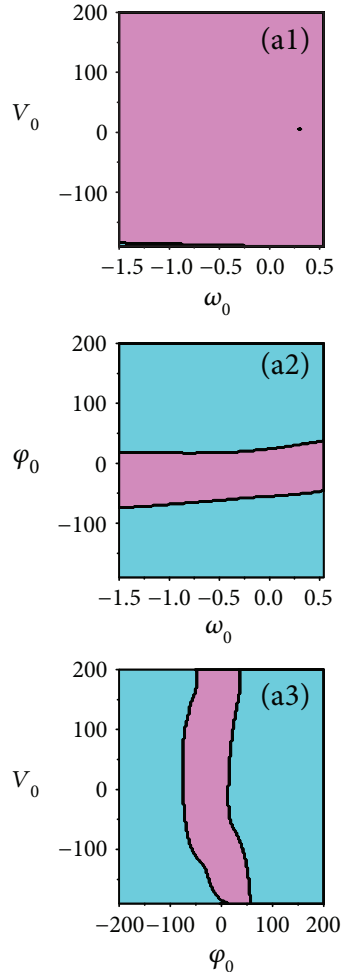
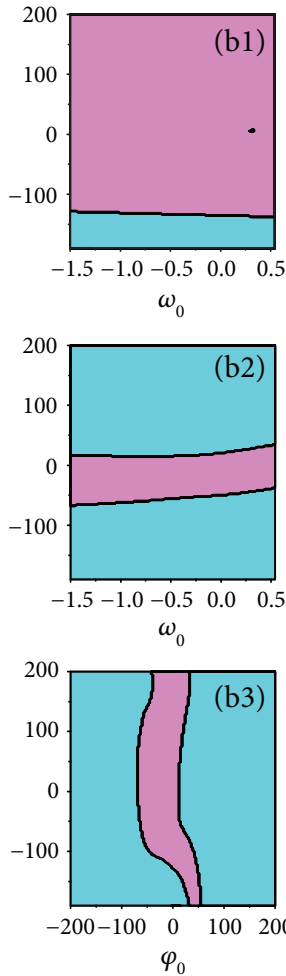
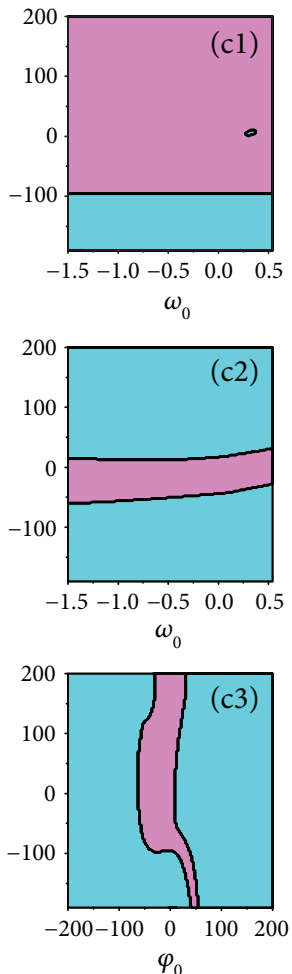
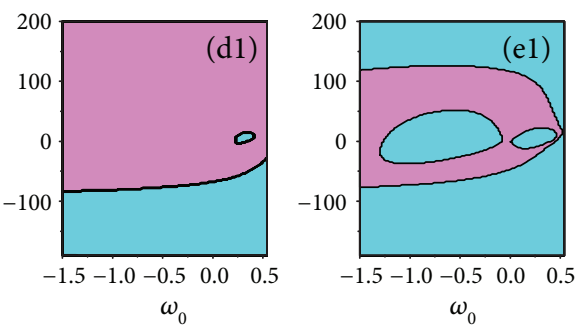

Quiescent
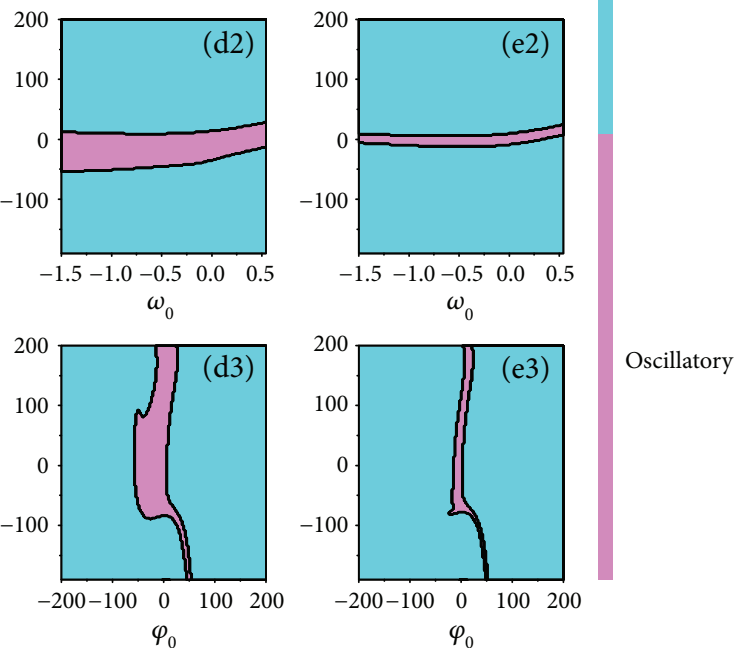

Figure 3: The regional distribution diagram of resting state and oscillating state under different initial values, for (a1-e1) $\varphi_{0}=0.1$ and $I_{\text {ext }}=40,50,60,70$, and 80; for (a2-e2) $V_{0}=80$ and $I_{\text {ext }}=40,50,60,70$, and 80; and for (a3-e3) $\omega_{0}=-1.5$ and $I_{\text {ext }}=40,50,60,70$, and 80.

TABLE 1: Summary of the type and position of bifurcation point.

\begin{tabular}{lcccccc}
\hline$k$ & & 0.1 & 0.2 & 0.4 & 0.5 & 0.6 \\
\hline \multirow{2}{*}{$\left.I_{\text {ext }}(\mu \mathrm{A})\right)$} & HOPF1 & -28.48 & -20.75 & -13.74 & -12.27 & -11.39 \\
& HOPF2 & 23.55 & 17.40 & 10.97 & 10.17 & 9.0 \\
\hline
\end{tabular}

reduced by the increase of the external stimulation current. Most interesting, the study found that the selection of the initial value will also be influenced by the induction coefficient $k$ under the same external stimulus current. This discovery is in accordance with the bifurcation diagram of Figure 2. Therefore, it is very important to choose the appropriate initial value with the different induction coefficients $k$. According to the initial value area shown in Figures 3 and 4, sampled time series for membrane potential and phase portraits are plotted in Figure 5.

The improved ML neural model can exhibit several kinds of oscillations. When the initial value is determined, the electric activity depends on the external forcing current. The change of electrical activity between the quiescent state and the spiking state can be observed by selecting different external forcing currents. The phase portraits of the external forcing currents $(\mu \mathrm{A})$ are chosen as $0,10,20,30,40$, and 80 in Figure 5(a). And Figures 5(b) and 5(c) show the time series of the membrane potential for $I_{\text {ext }}=0,40$, and 80 .

The numerical results in Figure 5(a) show that the regions of limit cycles become larger with increasing the external forcing current. There exist some thresholds in the system, which determines the conversion of the discharge mode of the neuron membrane potential. In the absence of external excitation, the neuron can still be discharged under the electromagnetic effect, as shown by the blue curve in Figure 5(b). With increasing the external forcing current, the amplitude of the neuron membrane potential will increase gradually; in other words, the amplitude of the periodic oscillation is related to the area size of the limit cycle. When the external forcing current is increased to a certain threshold, the system will change from the oscillating state to the resting state, as depicted in Figure 5(c).

Meanwhile, we studied the collective behavior of the two neurons driven by the excitatory autapse and inhibitory autapse in the case of electric coupling. The initial values are $V_{0}=100 \mathrm{mV}, \omega_{0}=-1.5 \mu \mathrm{A}$, and $\varphi_{0}=0.1$, and the external forcing current $I_{\text {ext }}=40 \mu \mathrm{A}$ is chosen for its simplicity; the induction coefficient is $k=0.1$. At first, the inter-spike interval of the $\beta$-neuron membrane potential (as in ISI representation) at different feedback gain $H_{\text {syn }}$ is calculated, and the results are plotted in Figures 6 and 7.

Presented results show clearly that feedback gain $H_{\text {syn }}$ as well as coupling strengths plays an important role in the modes of electrical activities. When the value of $H_{\text {syn }}$ is smaller, the rich discharge modes are observed in Figure 6. The value of the bifurcation point is also affected by the coupling intensity. It is found that the mode of $\beta$-neuron discharge does not exhibit periodicity, unless larger feedback gain could be applied. In essence, there may be two types of autapse in the system, and the increase of $H_{\text {syn }}$ makes the difference in the discharge of the two neurons increasing; that is, 

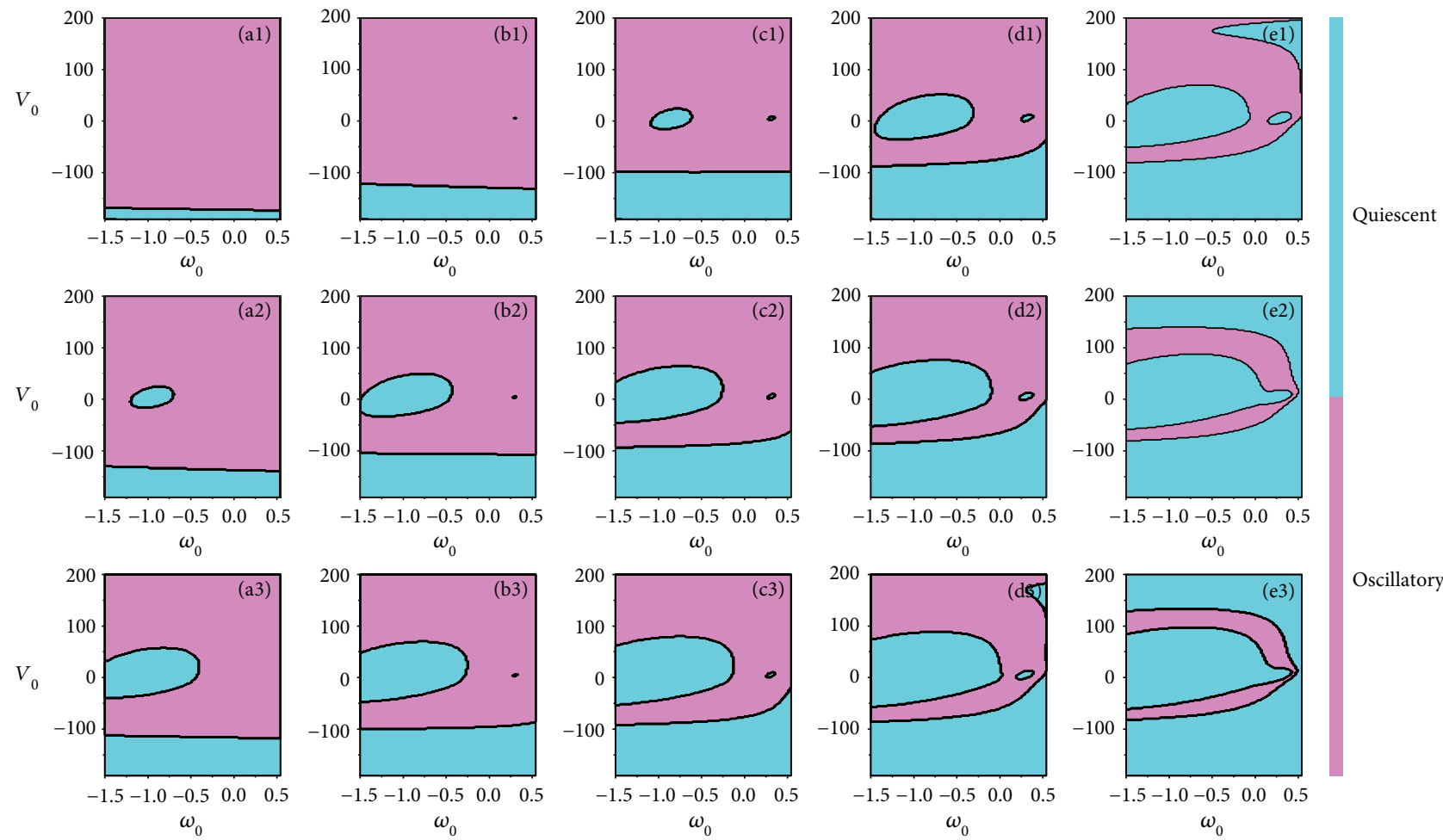

FIgure 4: The regional distribution diagram of the resting state and oscillating state under different initial values at $\varphi_{0}=0.1$, for $(\mathrm{a} 1-\mathrm{e} 1)$ $k=0.2$ and $I_{\mathrm{ext}}=30,40,50,60$, and 70; for (a2-e2) $k=0.3$ and $I_{\mathrm{ext}}=30,40,50,60$, and 70; and for (a3-e3) $k=0.4$ and $I_{\mathrm{ext}}=30,40,50$, 60 , and 70 .
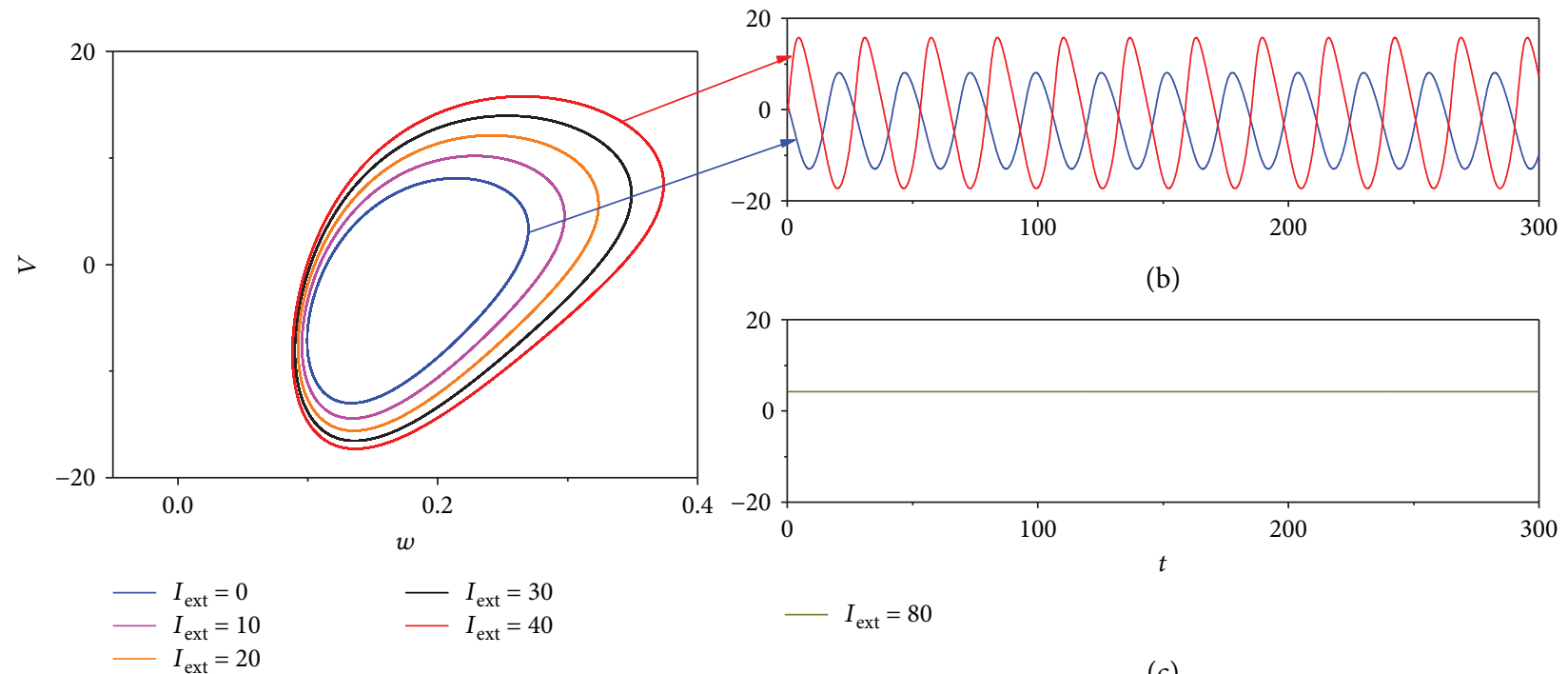

(b)

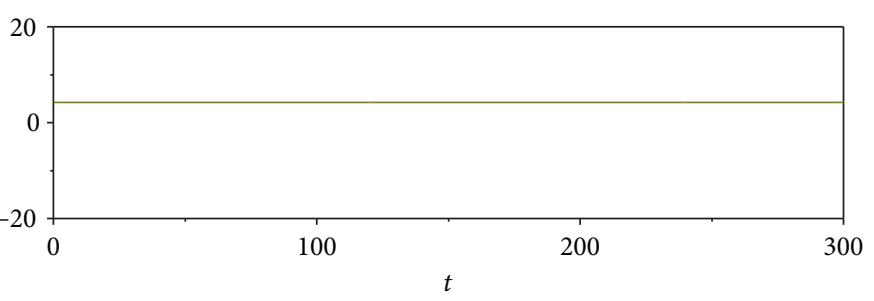

(a)

(c)

FIGURE 5: Sampled time series for membrane potential and phase portraits when different currents are calculated at the initial values $V_{0}=100$, $\omega_{0}=-0.1$, and $\varphi_{0}=0.1$, and the induction coefficient is $k=0.1$.

the inhibitory neurons tend to be quiescent and hence cannot affect the excitatory neurons. However, it can be observed that the modes of electrical activities depend significantly on the values of the synaptic feedback gain.
Within a certain coupling strength, if the error $e$ tends to zero with the time increased, the coupled neurons are fully synchronized. According to the analysis of the results of Figure 6, the sampled time series for membrane potential 


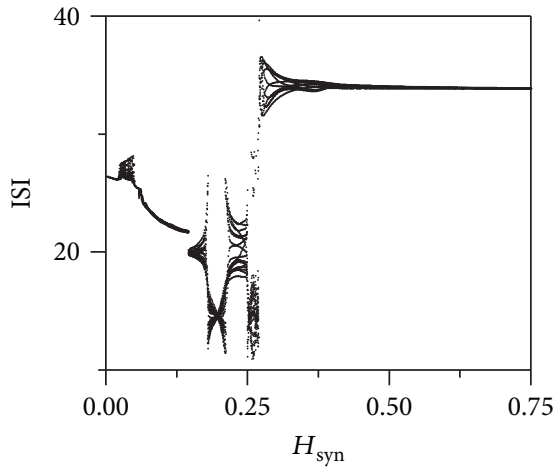

(a)

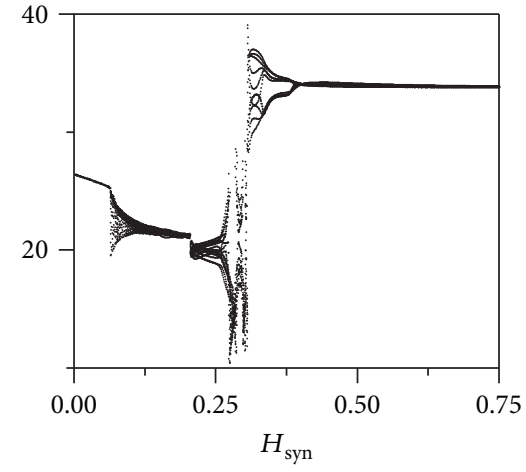

(b)

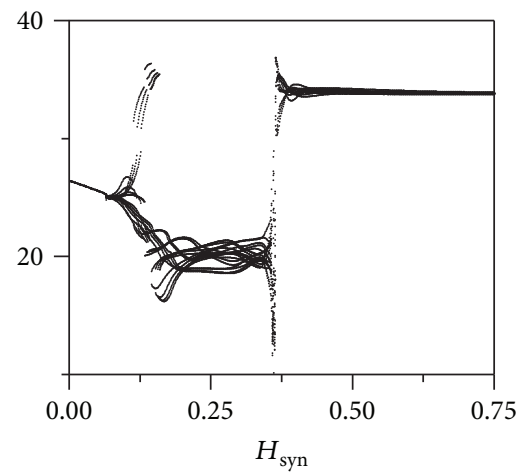

(c)

FIGURE 6: Bifurcation diagram associated with feedback gain $H_{\text {syn }}$ for different coupling strengths. The time lag $\tau=50,(\mathrm{a}) C=0.1 ;(\mathrm{b}) C=0.5$; and (c) $C=1$

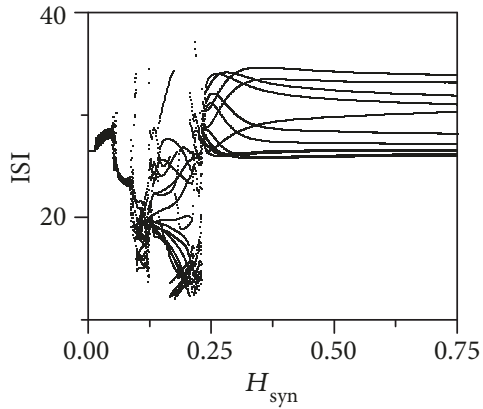

(a)

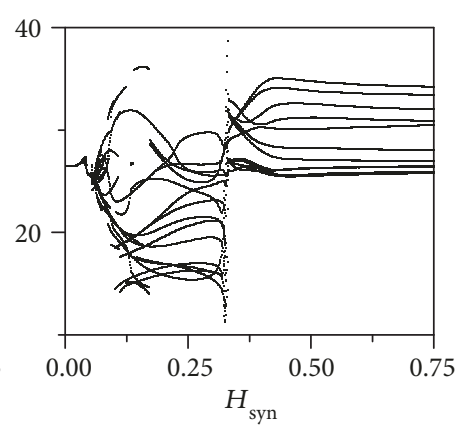

(b)

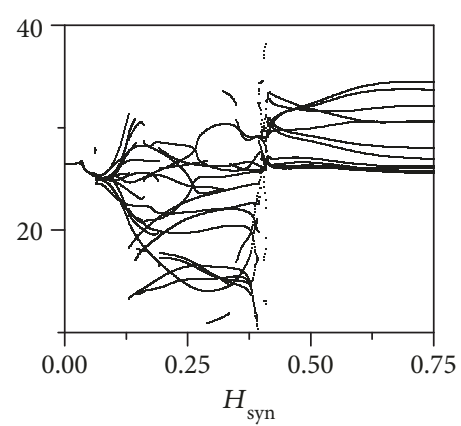

(c)

Figure 7: Bifurcation diagram associated with feedback gain $H_{\text {syn }}$ for different coupling strengths. The time lag $\tau=100$, (a) $C=0.1$; (b) $C=0.5$; and (c) $C=1$.

and phase portraits of the coupling strength $C=0.5$ are calculated with different feedback gains $H_{\text {syn }}$; the results are plotted in Figure 8.

The phase portraits and time series of the membrane potential of two coupled neurons are illustrated in Figure 8. The limit cycle is shown in Figure 8(a1), indicating that the $\beta$-neuron is periodic discharge. The region of the two limit cycles is different in Figure 8, (a2), and the value of the $V$ is within the range of $-20 \mathrm{mV}$ to $20 \mathrm{mV}$. The phase portrait of $\left(V_{\beta}, V_{\alpha}\right)$ is located near the corner line of the first quadrant, which means the occurrence of approximate synchronization. The error $e$ (blue line) is found to exhibit periodic oscillations in Figure 8, (a3). Interestingly, when the coupling strength $C$ is further increased, the phase portrait of $\left(V_{\beta}, V_{\alpha}\right)$ tends to have a straight line, and the results are shown in Figure 9.

The results in Figure 9 confirmed that the phase portrait of $\left(V_{\beta}, V_{\alpha}\right)$ coincides with a straight line, which is located on the angle bisector of the first quadrant. With appropriate time lag and feedback gain, the two coupled neuron system will synchronize with the increase of the coupling strength between neurons. For the improved model in this paper, the numerical results show that phase synchrony can be achieved by selecting the appropriate coupling intensity of 


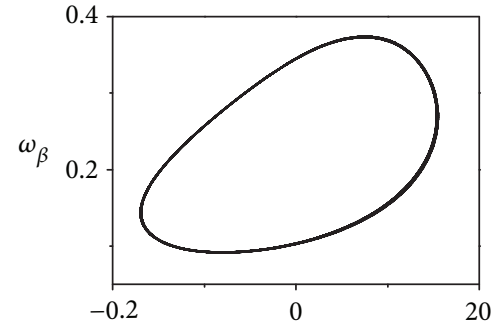

(a1)

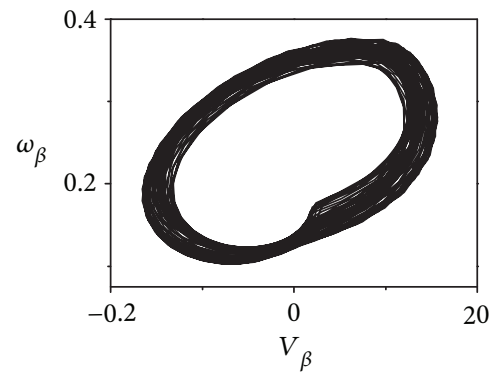

(a2)

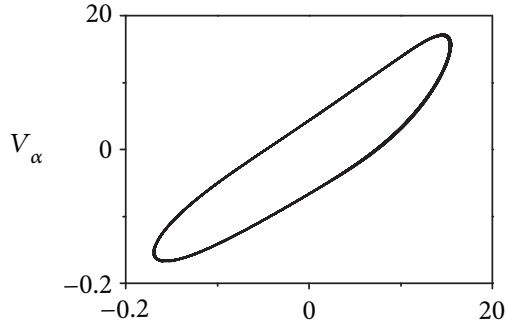

(b1)

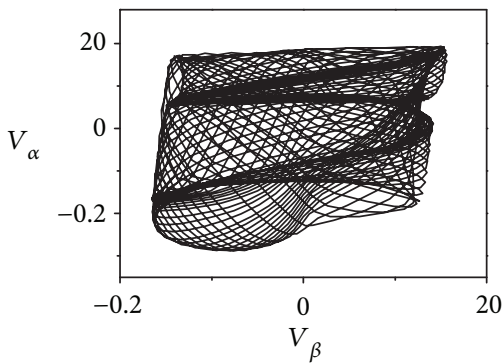

(b2)

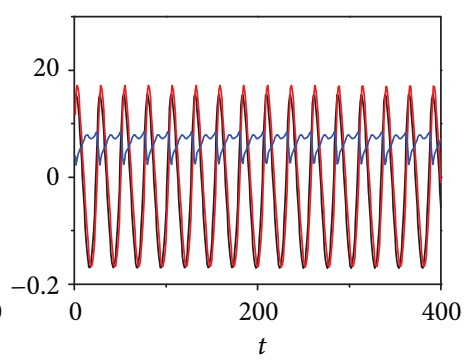

(c1)

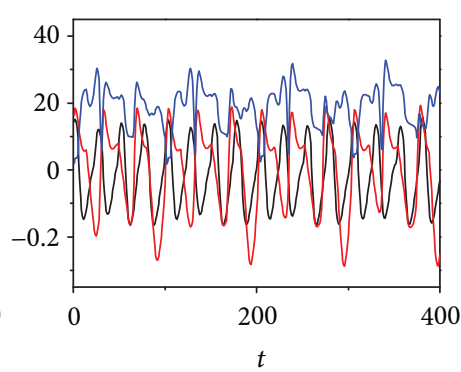

(c2)

FIGURE 8: Numerical simulation of the two neuron system. The parameters are chosen as $C=0.5$ and $\tau=50$ ms. (a1) The phase portrait of $\left(V_{\beta}, \omega_{\beta}\right), H_{\text {syn }}=0.03$. (a2) The phase portrait of $\left(V_{\beta}, V_{\alpha}\right), H_{\text {syn }}=0.03$. (a3) The time series of $V_{\beta}$ (black line), $V_{\alpha}$ (red line), and $e$ (blue line), $H_{\text {syn }}=0.03$. (b1) The phase portrait of $\left(V_{\beta}, \omega_{\beta}\right), H_{\text {syn }}=0.32$. (b2) The phase portrait of $\left(V_{\beta}, V_{\alpha}\right), H_{\text {syn }}=0.32$. (b3) The time series of $V_{\beta}$ (black line), $V_{\alpha}$ (red line), and $e$ (blue line), $H_{\text {syn }}=0.32$.

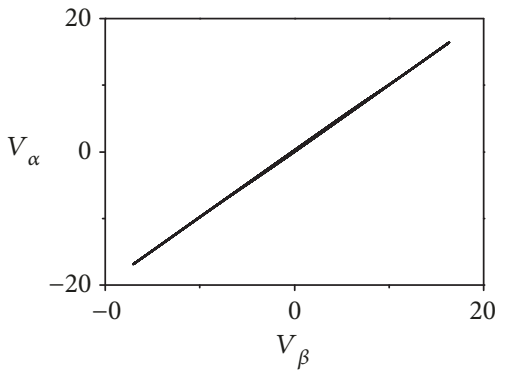

(a)

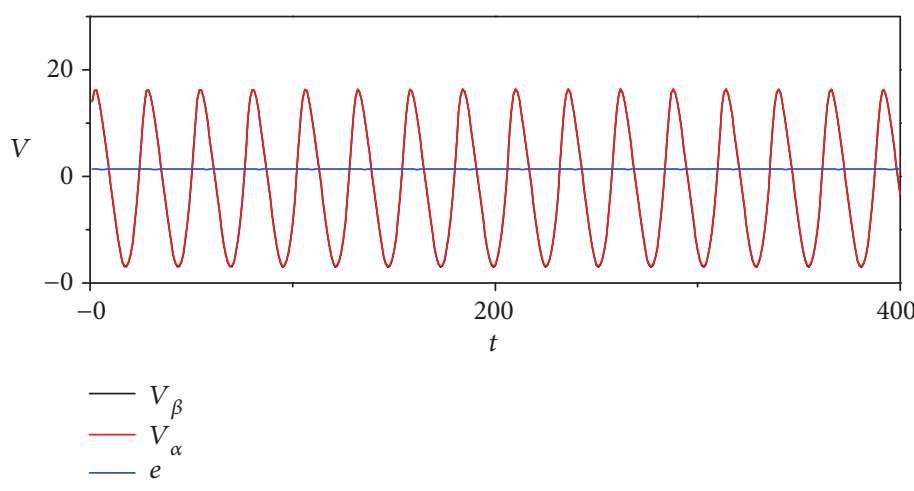

(b)

FIGURE 9: Phase portraits and sampled time series of membrane potential are calculated at coupling strength $C=20$, feedback gain $H_{\text {syn }}=0.03$, and time lag $\tau=50 \mathrm{~ms}$.

the two neurons with electromagnetic radiation. The effect of time lag in autapse should also be considered; for example, the time lag is increased to $100 \mathrm{~ms}$, and some results are found in Figures 7 and 10.

Results presented in Figure 7 reveal that the oscillatory pattern is largely influenced by the synaptic delay. For suitably long values of $\tau$, a complex oscillatory pattern can be observed. Interestingly, however, if the $H_{\text {syn }}$ is sufficiently high, we can observe the emergence of a periodic firing, which implies that there is a transition from chaos to periodic discharge in the system. Theoretically, under sufficiently long synaptic delay condition, neurons have enough time to fire more than once during a whole periodic cycle, before the synaptic currents caused by the first synchronous spiking within the same periodic cycle start to affect their firing [62].

It is found in Figure 10 that spiking and bursting discharge behaviors of $\beta$-neuron reappear depending on the gain and delay of the autapse. The error $e$ is observed to increase obviously through the time lag from $50 \mathrm{~ms}$ to $100 \mathrm{~ms}$. But what is more interesting is that the increment of $\tau$ makes the amplitude of the membrane potential of $\alpha$ neuron (excitatory) decrease in Figure 10, (a3). This trend may be that the role of the autapse is suppressed in the appropriate feedback gain and time lag. Therefore, the time lag plays a crucial role in the dynamics of the coupled system. Numerical studies on the synchronization of the two coupled 


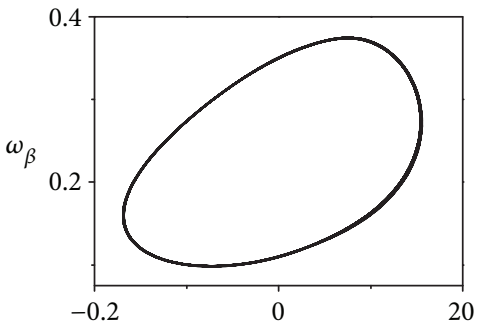

(a1)

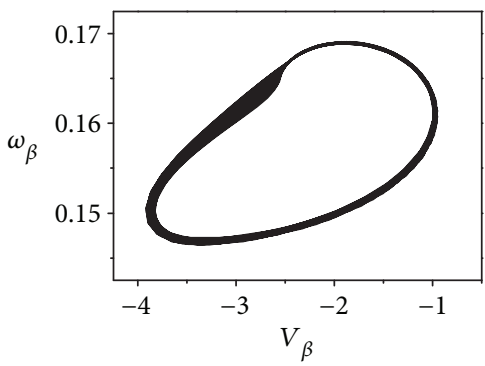

(b1)

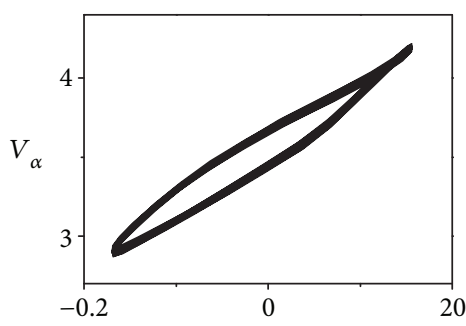

(a2)

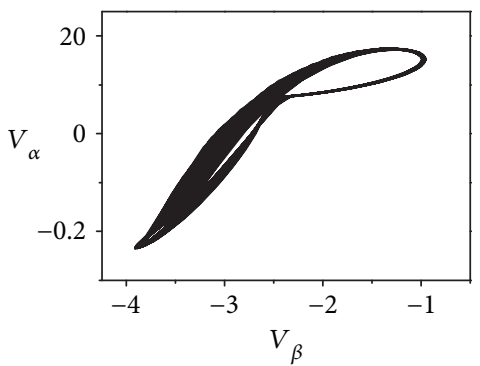

(b2)

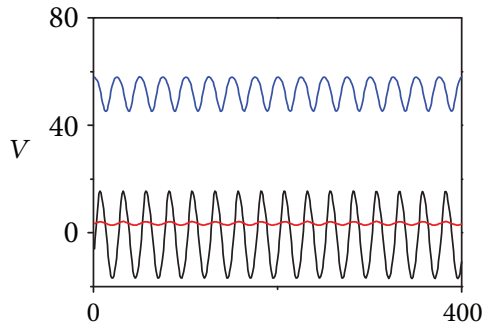

(a3)

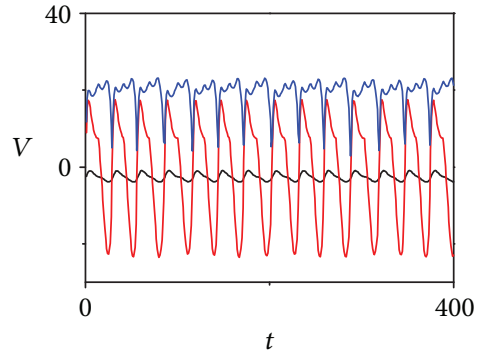

(c3)

FIgURE 10: Numerical simulations of the two neuron system. the parameters are chosen as $C=0.5$ and $\tau=100 \mathrm{~ms}$. (a1) The phase portrait of $\left(V_{\beta}, \omega_{\beta}\right), H_{\text {syn }}=0.1$. (a2) The phase portrait of $\left(V_{\beta}, V_{\alpha}\right), H_{\text {syn }}=0.1$. (a3) The time series of $V_{\beta}$ (black line), $V_{\alpha}$ (red line), and $e$ (blue line), $H_{\text {syn }}=0.1$. (b1) The phase portrait of $\left(V_{\beta}, \omega_{\beta}\right), H_{\text {syn }}=0.4$. (b2) The phase portrait of $\left(V_{\beta}, V_{\alpha}\right), H_{\text {syn }}=0.4$. (b3) The time series of $V_{\beta}$ (black line), $V_{\alpha}$ (red line), and $e$ (blue line), $H_{\text {syn }}=0.4$.

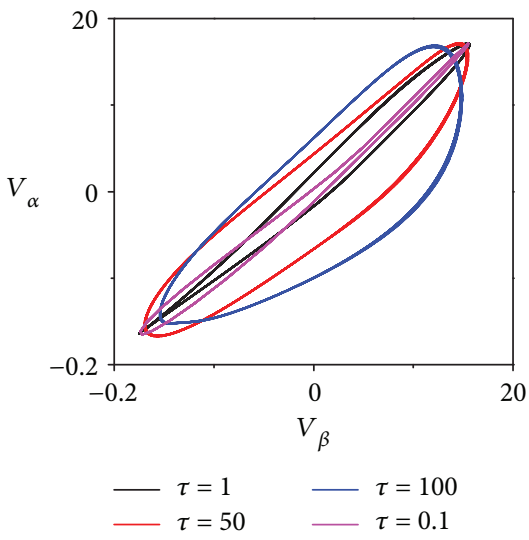

(a)

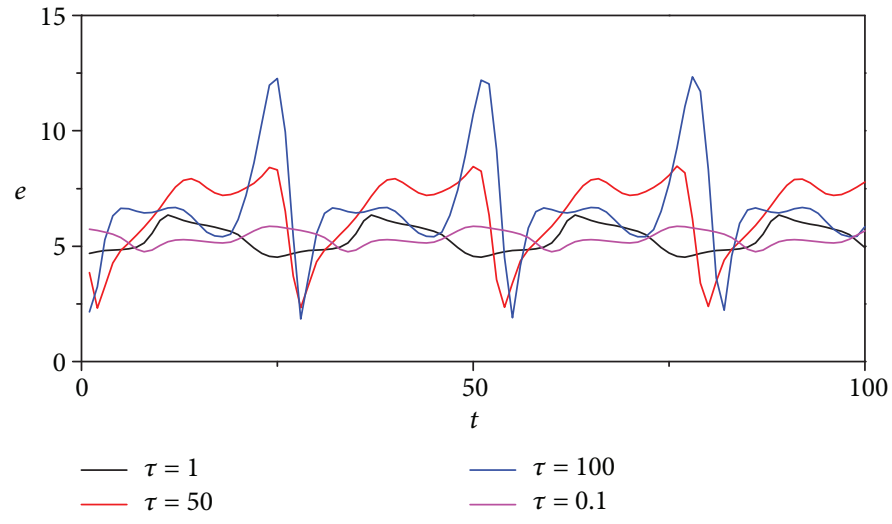

(b)

Figure 11: Phase portraits and error $e$ when different time-lag are calculated at $H_{\text {syn }}=0.03$, and the coupling strength $C=0.5$.

neuron system are affected with time lag, and the results are shown in Figure 11.

The trajectories when $H_{\text {syn }}=0.03$ indicate that the system of the two coupled neuron system is in the oscillation in Figure 11. When the time lag is 0.1, the membrane potential error $e$ of the neurons is smaller. Although the electrical coupling plays a dominant role in the synchronization of the system, the feedback gain from the synapse and time lag are equally important. The increment of time lag is not conducive to the synchronization of the coupling neuron system. This conclusion is consistent with the result of Figure 10.

\section{Conclusions}

In this paper, the dynamics of the improved Morris-Lecar neuron model under electromagnetic induction were investigated using bifurcation diagrams and time series of discharge; phase portraits of the neuron under different conditions are investigated in a numerical manner as well. By analyzing the simple numerical simulation of the improved model, the basic dynamic behaviors are obtained by introducing an external forcing current. In the case of the electromagnetic induction, the mechanism of neuron firing has been changed. That is, two Hopf bifurcation points 
are found with changing the external forcing current. Comparing these results with a previous work $[33,58]$, the bifurcation diagram has an obvious difference due to the consideration of electromagnetic induction based on the ML neural model. In fact, the fluctuation in membrane potential and signal propagation in the neuronal system can generate an induced electrical field and additive current in the media due to electromagnetic induction. As a result, the membrane potential of a neuron can be adjusted slightly by induction field and induced current associated with the variation of magnetic flux. By analyzing the interspike interval series of neural firing, we find that the improved model can generate electrical activity with multiple modes. These results are consistent with the observation observed in the experiment [8]. Meanwhile, the preliminary synchronization analysis of a system of excitatory and inhibitory neurons was conducted. In this aspect, it was unveiled that the neurons in the system can be synchronized by selecting an appropriate coupling strength. A longer time lag is not conducive to the system synchronization, and the higher the feedback gain $H_{\text {syn }}$ and the longer the time lag $\tau$ are, the more obvious the electrical mode changes in the two coupled neuron system; this conclusion is in accordance with previous experiments [63]. Synchronization phenomena are associated with either brain functions [64] or pathological brain states in the neural system. For example, Stam and Bruin used synchronization likelihood to characterize statistical interdependencies between EEG and MEG (magneto encephalography) signals in early and mild Alzheimer's disease $[65,66]$. Rubchinsky et al. [67] presented extensive experimental documentation of the relevance of synchronized oscillations to motor behavior in Parkinson's disease, and they confirmed that the real pathological state is not completely synchronous but showed a complex weak synchronization and highly intermittent dynamics. These results could provide potential theoretical supports for the treatment of neurological diseases.

\section{Data Availability}

The data used to support the findings of this study are available from the corresponding author upon request.

\section{Conflicts of Interest}

The authors declare that they have no conflicts of interest.

\section{Acknowledgments}

The authors gratefully thank Professor Jun Ma from the Lanzhou University of Technology for his constructive suggestions. This work was supported by the National Natural Science Foundation of China, under Grant nos. 11775091, 11474117 , and 11605014.

\section{References}

[1] R. FitzHugh, "Impulses and physiological states in theoretical models of nerve membrane," Biophysical Journal, vol. 1, no. 6, pp. 445-466, 1961.
[2] C. Morris and H. Lecar, "Voltage oscillations in the barnacle giant muscle fiber," Biophysical Journal, vol. 35, no. 1, pp. 193-213, 1981.

[3] J. L. Hindmarsh and R. M. Rose, "A model of the nerve impulse using two first-order differential equations," Nature, vol. 296, no. 5853, pp. 162-164, 1982.

[4] M. Storace, D. Linaro, and E. de Lange, "The Hindmarsh-Rose neuron model: an bifurcation analysis and piecewise-linear approximations," Chaos: An Interdisciplinary Journal of Nonlinear Science, vol. 18, no. 3, article 033128, 2008.

[5] J. Nagumo and S. Sato, "On a response characteristic of a mathematical neuron model," Kybernetik, vol. 10, no. 3, pp. 155-164, 1972.

[6] H. R. Wilson and J. D. Cowan, "Excitatory and inhibitory interactions in localized populations of model neurons," Biophysical Journal, vol. 12, no. 1, pp. 1-24, 1972.

[7] A. L. Hodgkin and A. F. Huxley, "The dual effect of membrane potential on sodium conductance in the giant axon of Loligo," The Journal of Physiology, vol. 116, no. 4, pp. 497-506, 1952.

[8] H. Gu, W. Ren, Q. Lu, S. Wu, M. Yang, and W. Chen, "Integer multiple spiking in neuronal pacemakers without external periodic stimulation," Physics Letters A, vol. 285, no. 1-2, pp. 63-68, 2001.

[9] M. T. M. Koper and B. D. Aguda, "Experimental demonstration of delay and memory effects in the bifurcations of nickel electrodissolution," Physical Review E, vol. 54, no. 1, pp. 960 963, 1996.

[10] H. Gu, "Experimental observation of transition from chaotic bursting to chaotic spiking in a neural pacemaker," Chaos: An Interdisciplinary Journal of Nonlinear Science, vol. 23, no. 2, p. 023126, 2013.

[11] K. Tsumoto, H. Kitajima, T. Yoshinaga, K. Aihara, and H. Kawakami, "Bifurcations in Morris-Lecar neuron model," Neurocomputing, vol. 69, no. 4-6, pp. 293-316, 2006.

[12] D. Golomb and J. Rinzel, "Clustering in globally coupled inhibitory neurons," Physica D: Nonlinear Phenomena, vol. 72, no. 3, pp. 259-282, 1994.

[13] L. M. Pecora and T. L. Carroll, "Paper 9 - Synchronization in chaotic systems," in Controlling Chaos, T. Kapitaniak, Ed., vol. 6, pp. 142-145, Academic Press, San Diego, CA, USA, 1996, http://www.sciencedirect.com/science/article/pii/B9780 123968401500400

[14] F. Varela, J. P. Lachaux, E. Rodriguez, and J. Martinerie, "The brainweb: phase synchronization and large-scale integration," Nature Reviews Neuroscience, vol. 2, no. 4, pp. 229-239, 2001.

[15] M. Bazhenov, R. Huerta, M. I. Rabinovich, and T. Sejnowski, "Cooperative behavior of a chain of synaptically coupled chaotic neurons," Physica D: Nonlinear Phenomena, vol. 116, no. 3-4, pp. 392-400, 1998.

[16] W. Zhang, Y. Tang, X. Wu, and J. A. Fang, "Synchronization of nonlinear dynamical networks with heterogeneous impulses," IEEE Transactions on Circuits and Systems I: Regular Papers, vol. 61, no. 4, pp. 1220-1228, 2014.

[17] N. Burić, K. Todorović, and N. Vasović, "Synchronization of bursting neurons with delayed chemical synapses," Physical Review E, vol. 78, no. 3, article 036211, 2008.

[18] Y. Xu, Y. Jia, J. Ma, T. Hayat, and A. Alsaedi, "Collective responses in electrical activities of neurons under field coupling," Scientific Reports, vol. 8, no. 1, p. 1349, 2018.

[19] C. Yao, M. Zhan, J. Shuai, J. Ma, and J. Kurths, "Insensitivity of synchronization to network structure in chaotic pendulum 
systems with time-delay coupling," Chaos, vol. 27, no. 12, article 126702, 2017.

[20] P. M. Gokul and T. Kapitaniak, "Synchronization in coupled multistable systems with hidden attractors," Mathematical Problems in Engineering, vol. 2017, Article ID 5214235, 6 pages, 2017.

[21] M. Zhan, G. Hu, and J. Yang, "Synchronization of chaos in coupled systems," Physical Review E, vol. 62, no. 2, pp. 29632966, 2000.

[22] W. Zou and M. Zhan, "Complete periodic synchronization in coupled systems," Chaos, vol. 18, no. 4, article 043115, 2008.

[23] G. Zhang, F. Wu, C. Wang, and J. Ma, "Synchronization behaviors of coupled systems composed of hidden attractors," International Journal of Modern Physics B, vol. 31, no. 26, article 1750180, 2017.

[24] Y. Xu, Y. Jia, J. Ma, A. Alsaedi, and B. Ahmad, "Synchronization between neurons coupled by memristor," Chaos, Solitons \& Fractals, vol. 104, pp. 435-442, 2017.

[25] M. Gosak, M. Marhl, and M. Perc, "Pacemaker-guided noiseinduced spatial periodicity in excitable media," Physica D: Nonlinear Phenomena, vol. 238, no. 5, pp. 506-515, 2009.

[26] A. A. M. Yousif, L. Lu, M. Ge, Y. Xu, and Y. Jia, "Effects of intrinsic and extrinsic noises on transposons kinetics," Chinese Physics B, vol. 27, no. 3, article 030501, 2018.

[27] Y. Xu, Y. Jia, M. Ge, L. Lu, L. Yang, and X. Zhan, "Effects of ion channel blocks on electrical activity of stochastic HodgkinHuxley neural network under electromagnetic induction," Neurocomputing, vol. 283, pp. 196-204, 2018.

[28] Q. Y. Wang, Q. S. Lu, G. R. Chen, and D. H. Guo, "Chaos synchronization of coupled neurons with gap junctions," Physics Letters A, vol. 356, no. 1, pp. 17-25, 2006.

[29] Y. Wu, J. Xu, and W. JinJ. Wang, X. Liao, and Z. Yi, "Synchronous behaviors of two coupled neurons," in Advances in Neural Networks - ISNN 2005. ISNN 2005, vol. 3496 of Lecture Notes in Computer Science, pp. 302-307, Springer, Berlin, Heidelberg, 2005.

[30] V.-T. Pham, C. Volos, S. Jafari, and T. Kapitaniak, "A novel cubic-equilibrium chaotic system with coexisting hidden attractors: analysis, and circuit implementation," Journal of Circuits, Systems and Computers, vol. 27, no. 4, article 1850066, 2018.

[31] G. Ren, J. Tang, J. Ma, and Y. Xu, "Detection of noise effect on coupled neuronal circuits," Communications in Nonlinear Science and Numerical Simulation, vol. 29, no. 1-3, pp. 170-178, 2015.

[32] M. Ozer and N. H. Ekmekci, "Effect of channel noise on the time-course of recovery from inactivation of sodium channels," Physics Letters A, vol. 338, no. 2, pp. 150-154, 2005.

[33] L. Xue, J. Wang, B. Deng, and F. Dong, "Inhibitory chemical coupling of electronic Morris-Lecar neuron model and its bifurcation analysis," in 2008 30th Annual International Conference of the IEEE Engineering in Medicine and Biology Society, pp. 2461-2464, Vancouver, BC, Canada, August 2008.

[34] M. Nouri, G. R. Karimi, A. Ahmadi, and D. Abbott, "Digital multiplierless implementation of the biological FitzHughNagumo model," Neurocomputing, vol. 165, pp. 468-476, 2015.

[35] E. Tlelo-Cuautle, L. G. de la Fraga, V.-T. Pham, C. Volos, S. Jafari, and A. d. J. Quintas-Valles, "Dynamics, FPGA realization and application of a chaotic system with an infinite number of equilibrium points," Nonlinear Dynamics, vol. 89, no. 2, pp. 1129-1139, 2017.
[36] S. Vaidyanathan, C. Volos, V.-T. Pham, K. Madhavan, and B. A. Idowu, "Adaptive backstepping control, synchronization and circuit simulation of a 3-D novel jerk chaotic system with two hyperbolic sinusoidal nonlinearities," Archives of Control Sciences, vol. 24, no. 3, pp. 375-403, 2014.

[37] S. Vaidyanathan and C. Volos, "Analysis and adaptive control of a novel 3-D conservative no-equilibrium chaotic system," Archives of Control Sciences, vol. 25, no. 3, pp. 333-353, 2015.

[38] S. T. Kingni, V.-T. Pham, S. Jafari, G. R. Kol, and P. Woafo, "Three-dimensional chaotic autonomous system with a circular equilibrium: analysis, circuit implementation and its fractional-order form," Circuits, Systems, and Signal Processing, vol. 35, no. 6, pp. 1933-1948, 2016.

[39] M. Conti and C. Turchetti, "Approximate identity neural networks for analog synthesis of nonlinear dynamical systems," IEEE Transactions on Circuits and Systems I: Fundamental Theory and Applications, vol. 41, no. 12, pp. 841-858, 1994.

[40] V.-T. Pham, C. Volos, S. Jafari, Z. Wei, and X. Wang, "Constructing a novel no-equilibrium chaotic system," International Journal of Bifurcation and Chaos, vol. 24, no. 5, p. 1450073, 2014.

[41] H. Akiyama, Y. Shimizu, H. Miyakawa, and M. Inoue, "Extracellular DC electric fields induce nonuniform membrane polarization in rat hippocampal CA1 pyramidal neurons," Brain Research, vol. 1383, pp. 22-35, 2011.

[42] C. Wang and J. Ma, "A review and guidance for pattern selection in spatiotemporal system," International Journal of Modern Physics B, vol. 32, no. 6, article 1830003, 2018.

[43] J. Ma and J. Tang, "A review for dynamics in neuron and neuronal network," Nonlinear Dynamics, vol. 89, no. 3, pp. 15691578, 2017.

[44] M. Lv, C. Wang, G. Ren, J. Ma, and X. Song, "Model of electrical activity in a neuron under magnetic flow effect," Nonlinear Dynamics, vol. 85, no. 3, pp. 1479-1490, 2016.

[45] K.-A. Hossmann and D. M. Hermann, "Effects of electromagnetic radiation of mobile phones on the central nervous system," Bioelectromagnetics, vol. 24, no. 1, pp. 49-62, 2003.

[46] A. E. Kaprana, A. D. Karatzanis, E. P. Prokopakis et al., "Studying the effects of mobile phone use on the auditory system and the central nervous system: a review of the literature and future directions," European Archives of Oto-Rhino-Laryngology, vol. 265, no. 9, pp. 1011-1019, 2008.

[47] L. Lu, Y. Jia, W. Liu, and L. Yang, "Mixed stimulus-induced mode selection in neural activity driven by high and low frequency current under electromagnetic radiation," Complexity, vol. 2017, Article ID 7628537, 11 pages, 2017.

[48] M. Ge, Y. Jia, Y. Xu, and L. Yang, "Mode transition in electrical activities of neuron driven by high and low frequency stimulus in the presence of electromagnetic induction and radiation," Nonlinear Dynamics, vol. 91, no. 1, pp. 515-523, 2018.

[49] L. Lulu, J. Ya, X. Ying, G. Mengyan, Y. Lijian, and Z. Xuan, "Energy dependence on modes of electric activities of neuron driven by different external mixed signals under electromagnetic induction," SCIENCE CHINA Technological Sciences, vol. 61, 2018.

[50] C. K. Volos, I. M. Kyprianidis, I. N. Stouboulos, and S. Vaidyanathan, "A new concept in synchronization of coupled neuromorphic circuits," Journal of Engineering Science and Technology Review, vol. 8, no. 2, pp. 157-173, 2015.

[51] L. O. Chua and S. M. Kang, "Memristive devices and systems," Proceedings of the IEEE, vol. 64, no. 2, pp. 209-223, 1976. 
[52] L. Shi, X. Yang, Y. Li, and Z. Feng, "Finite-time synchronization of nonidentical chaotic systems with multiple timevarying delays and bounded perturbations," Nonlinear Dynamics, vol. 83, no. 1-2, pp. 75-87, 2016.

[53] F. Wu, C. Wang, Y. Xu, and J. Ma, "Model of electrical activity in cardiac tissue under electromagnetic induction," Scientific Reports, vol. 6, no. 1, p. 28, 2016.

[54] L. Chua, "Memristor-the missing circuit element," IEEE Transactions on Circuit Theory, vol. 18, no. 5, pp. 507-519, 1971.

[55] D. Somers and N. Kopell, "Rapid synchronization through fast threshold modulation,” Biological Cybernetics, vol. 68, no. 5, pp. 393-407, 1993.

[56] I. Belykh, E. de Lange, and M. Hasler, "Synchronization of bursting neurons: what matters in the network topology," Physical Review Letters, vol. 94, no. 18, article 188101, 2005.

[57] C. A. S. Batista, R. L. Viana, F. A. S. Ferrari, S. R. Lopes, A. M. Batista, and J. C. P. Coninck, "Control of bursting synchronization in networks of Hodgkin-Huxley-type neurons with chemical synapses," Physical Review E, vol. 87, no. 4, article 042713, 2013.

[58] Y. Tao, H. Gu, and X. Ding, "Spatial coherence resonance and spatial pattern transition induced by the decrease of inhibitory effect in a neuronal network," International Journal of Modern Physics B, vol. 31, no. 26, article 1750179, 2017.

[59] Q. Wang, H. Zhang, M. Perc, and G. Chen, "Multiple firing coherence resonances in excitatory and inhibitory coupled neurons," Communications in Nonlinear Science and Numerical Simulation, vol. 17, no. 10, pp. 3979-3988, 2012.

[60] Y. X. Fu, Y. M. Kang, and Y. Xie, "Subcritical Hopf bifurcation and stochastic resonance of electrical activities in neuron under electromagnetic induction," Frontiers in Computational Neuroscience, vol. 12, p. 6, 2018.

[61] Q. Zhang, Bifurcation and Chaos Theory and Application, Tianjin University Press, Tianjin, 2015.

[62] D. Guo, Q. Wang, and M. Perc, "Complex synchronous behavior in interneuronal networks with delayed inhibitory and fast electrical synapses," Physical Review E, vol. 85, no. 6, pp. 878896, 2012.

[63] J. Ma, X. Song, W. Jin, and C. Wang, "Autapse-induced synchronization in a coupled neuronal network," Chaos, Solitons \& Fractals, vol. 80, pp. 31-38, 2015.

[64] A. Riehle, S. Grün, M. Diesmann, and A. Aertsen, "Spike synchronization and rate modulation differentially involved in motor cortical function," Science, vol. 278, no. 5345, pp. 1950-1953, 1997.

[65] J. J. Palop, J. Chin, and L. Mucke, "A network dysfunction perspective on neurodegenerative diseases," Nature, vol. 443, no. 7113, pp. 768-773, 2006.

[66] C. J. Stam and E. A. de Bruin, "Scale-free dynamics of global functional connectivity in the human brain," Human Brain Mapping, vol. 22, no. 2, pp. 97-109, 2004.

[67] L. L. Rubchinsky, C. Park, and R. M. Worth, "Intermittent neural synchronization in Parkinson's disease," Nonlinear Dynamics, vol. 68, no. 3, pp. 329-346, 2012. 


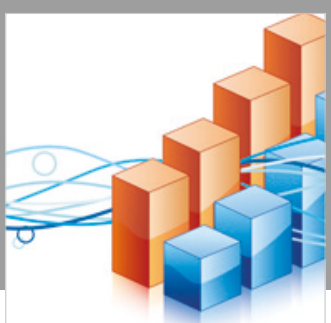

Advances in

Operations Research

\section{-n-m}
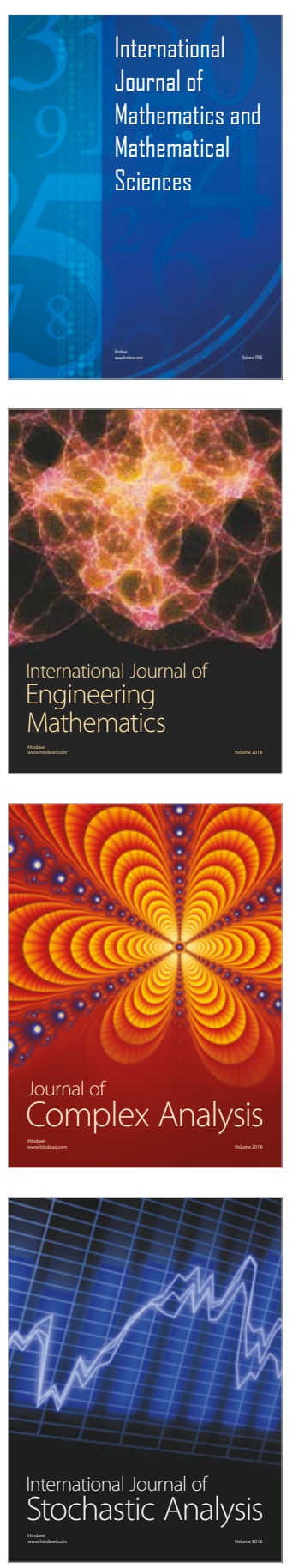
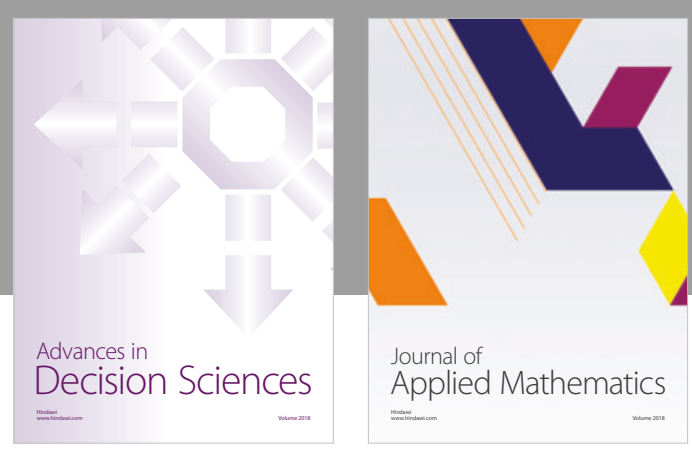

Journal of

Applied Mathematics
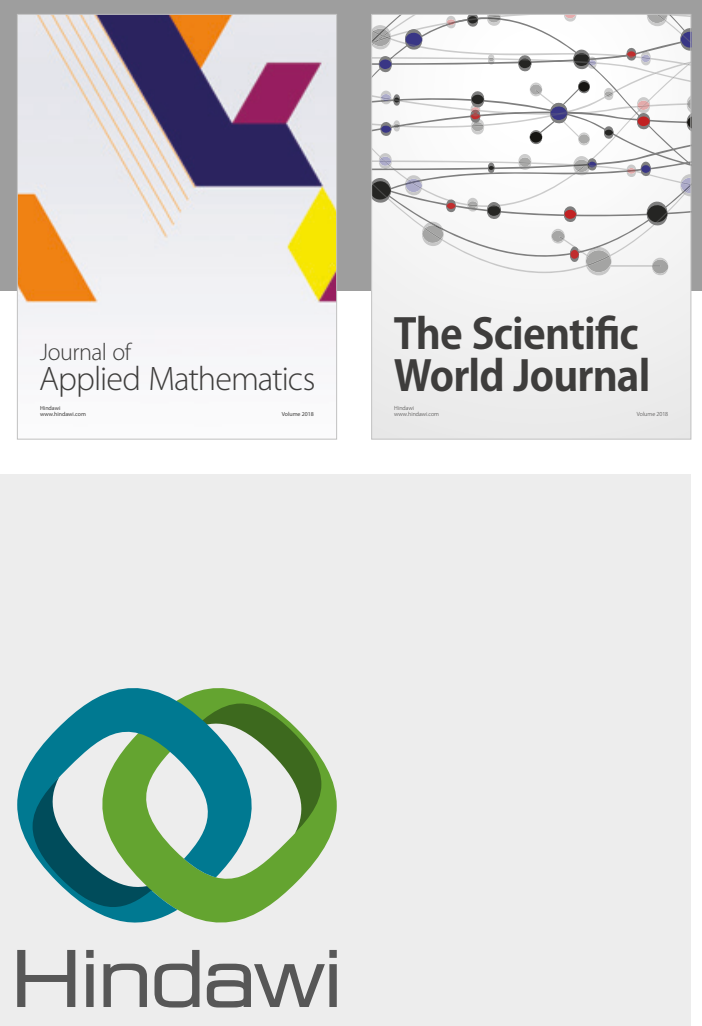

Submit your manuscripts at

www.hindawi.com

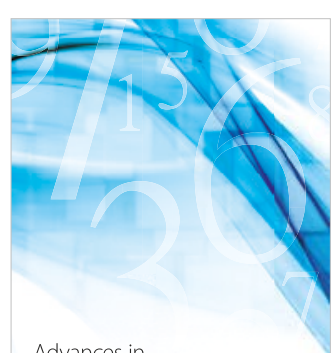

Advances in
Numerical Analysis
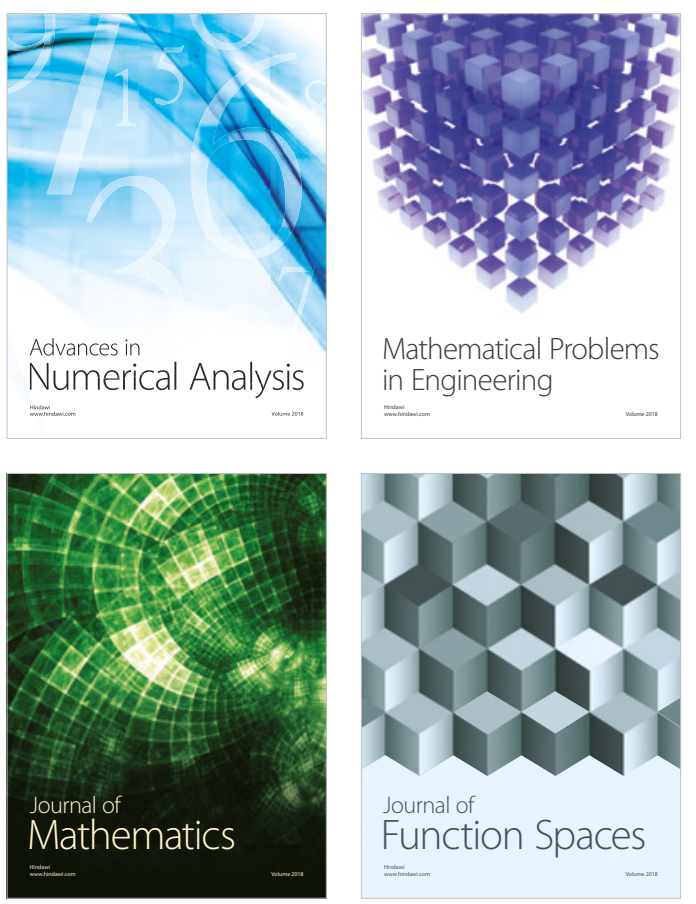

Mathematical Problems in Engineering

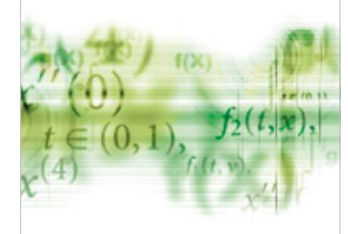

International Journal of

Differential Equations

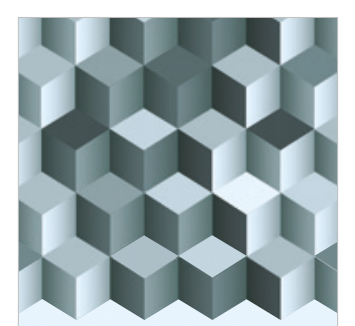

Journal of

Function Spaces
The Scientific

World Journal

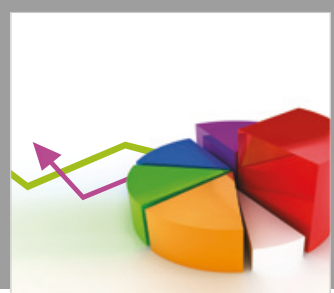

Journal of

Probability and Statistics
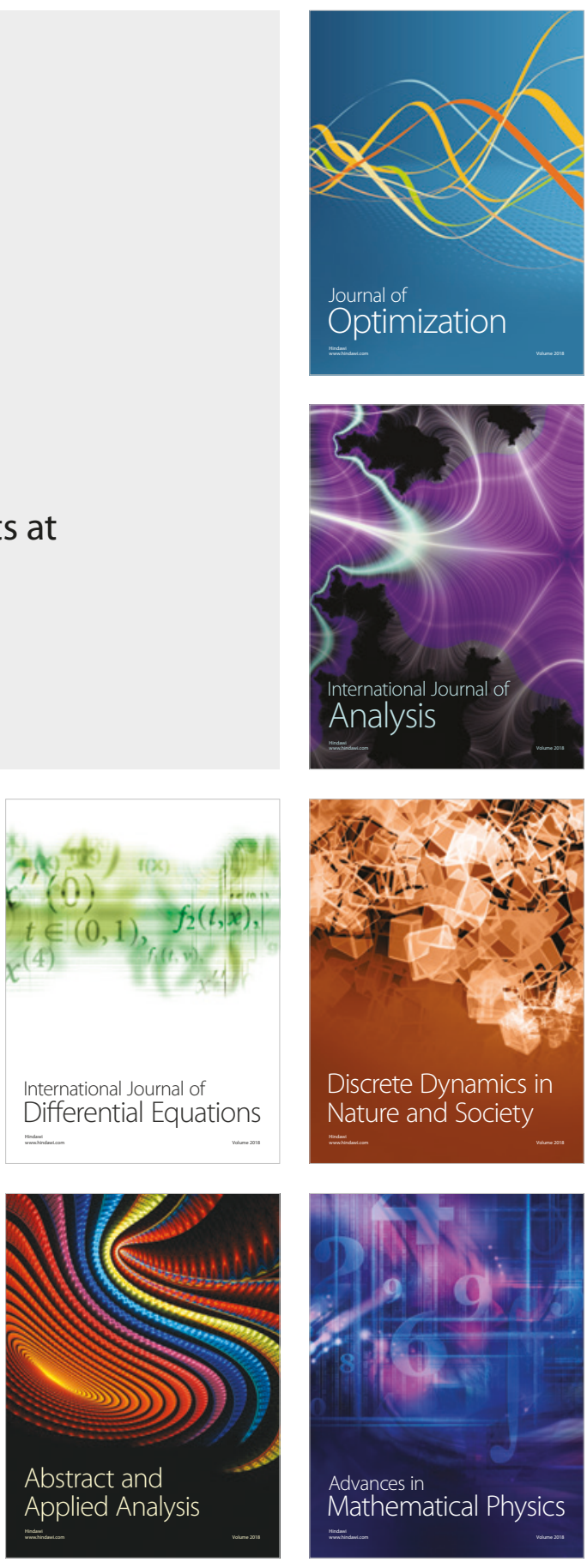